\title{
Proving uniqueness for the solution of the problem of homogeneous and anisotropic micropolar thermoelasticity
}

\author{
Adina Chirilă ${ }^{1}$, Ravi P Agarwal ${ }^{2}$ and Marin Marin ${ }^{1 *}$ (D)
}

\section{${ }^{\text {*Correspondence: }}$}

m.marin@unitbv.ro

${ }^{1}$ Department of Mathematics,

Transilvania University of Braşov,

Braşov, Romania

Full list of author information is

available at the end of the article

\section{Introduction}

In [1], the author derives some uniqueness criteria for solutions of the Cauchy problem for the standard equations of dynamical linear thermoelasticity backward in time. LagrangeBrun identities are combined with some differential inequalities in order to show that the final boundary value problem associated with the linear thermoelasticity backward in time has at most one solution in appropriate classes of displacement-temperature fields. The uniqueness results are obtained under the assumptions that the density mass and the specific heat are strictly positive and the conductivity tensor is positive definite.

According to [2], in a micropolar continuum the deformation is described not only by the displacement vector, but also by an independent rotation vector. This rotation vector specifies the orientation of a triad of director vectors attached to each material particle. A material point can experience a microrotation without undergoing a macrodisplacement.

The results obtained in this paper are extensions of those for the solutions of the Cauchy problem for the standard equations of dynamical linear thermoelasticity. The identities established in this paper lay the foundations for a uniqueness result.

In [3], Eringen establishes a uniqueness theorem for the boundary initial value problem of linear micropolar elastodynamics by means of some relations between the kinetic density, the strain energy density and the total power of the applied forces.

The spatial and the time arguments of a function will be omitted when there is no likelihood of confusion. A superposed dot denotes differentiation with respect to time $t$ and a subscript preceded by a comma denotes differentiation with respect to the corresponding spatial variable. The subscripts $i, j, k, m, n$ take values $1,2,3$ and summation is implied by index repetition.

Let $\bar{B}$ denote a regular region of the three dimensional Euclidean space occupied by a homogeneous micropolar body whose boundary is $\partial B$. The interior of $\bar{B}$ is denoted by $B$.

(c) The Author(s) 2017. This article is distributed under the terms of the Creative Commons Attribution 4.0 International License (http://creativecommons.org/licenses/by/4.0/), which permits unrestricted use, distribution, and reproduction in any medium, provided you give appropriate credit to the original author(s) and the source, provide a link to the Creative Commons license, and indicate if changes were made. 
The governing equations of the theory of anisotropic and homogeneous micropolar thermoelasticity, as described in [4] and also in [5-8] and [9], are the equations of motion

$$
t_{i j, j}+\rho F_{i}=\rho \ddot{u}_{i}, \quad m_{i j, j}+\varepsilon_{i j k} t_{j k}+\rho M_{i}=I_{i j} \ddot{\varphi}_{j},
$$

and the equation of energy

$$
\rho \dot{\eta}=\frac{\rho}{\theta_{0}} r-q_{i, i}
$$

Equations (1) and (2) are defined for $(x, t) \in B \times[0, \infty)$.

When the reference solid has a center of symmetry at each point, but is otherwise nonisotropic, then the constitutive equations, defined for $(x, t) \in \bar{B} \times[0, \infty)$, are

$$
\begin{aligned}
& t_{i j}=A_{i j m n} \varepsilon_{m n}+B_{i j m n} \gamma_{m n}-D_{i j} \theta, \\
& m_{i j}=B_{m n i j} \varepsilon_{m n}+C_{i j m n} \gamma_{m n}-E_{i j} \theta, \\
& \rho \eta=D_{i j} \varepsilon_{i j}+E_{i j} \gamma_{i j}+\frac{c}{\theta_{0}} \theta, \\
& q_{i}=-\frac{1}{\theta_{0}} K_{i j} \beta_{j} .
\end{aligned}
$$

The deformation tensors $\varepsilon_{i j}$ and $\gamma_{i j}$ used in equations (3) are defined in $\bar{B} \times[0, \infty)$ by means of the geometric equations

$$
\varepsilon_{i j}=u_{j, i}+\varepsilon_{j i k} \varphi_{k}, \quad \gamma_{i j}=\varphi_{j, i} .
$$

The system of equations is complete if we add the law of heat flow

$$
\beta_{i}=\theta_{, i}
$$

for all $(x, t) \in \bar{B} \times[0, \infty)$.

In the equations above we used the following notations: $u_{i}$ are the components of the displacement vector, $\varphi_{i}$ are the components of the microrotation vector, $t_{i j}$ are the components of the stress tensor, $m_{i j}$ are the components of the couple stress tensor, $q_{i}$ are the components of the heat conduction vector, $\eta$ is the specific entropy per unit mass, $\rho$ is the constant reference density, $\theta_{0}$ is the constant reference temperature, $\theta$ is the temperature measured from the temperature $\theta_{0}, c$ is the specific heat, $I_{i j}$ are the components of the inertia, $\beta_{i}$ are the components of the thermal displacement gradient vector, $F_{i}$ are the components of the external body force vector, $M_{i}$ are the components of the external body couple vector, $r$ is the external rate of heat supply per unit mass and $\varepsilon_{i j k}$ is the alternating symbol.

The coefficients from (1) and (3), that is, $A_{i j m n}, B_{i j m n}, C_{i j m n}, D_{i j}, E_{i j}, I_{i j}$, $K_{i j}$, and $c$ are constant constitutive coefficients subject to the following symmetry conditions:

$$
A_{i j m n}=A_{m n i j}, \quad C_{i j m n}=C_{m n i j}, \quad I_{i j}=I_{j i}, \quad K_{i j}=K_{j i} .
$$


We further assume that $c_{0} \geq 0$ and $K_{i j} \theta_{i} \theta_{j} \geq 0$.

The free energy $\Psi$, used to obtain the constitutive equations, is given by

$$
\begin{aligned}
\rho \Psi= & \frac{1}{2} A_{i j m n} \varepsilon_{i j} \varepsilon_{m n}+B_{i j m n} \varepsilon_{i j} \gamma_{m n}+\frac{1}{2} C_{i j m n} \gamma_{i j} \gamma_{m n} \\
& -D_{i j} \varepsilon_{i j} \theta-E_{i j} \gamma_{i j} \theta-\frac{c}{2 \theta_{0}} \theta^{2}+\frac{c}{2 \theta_{0}} K_{i j} \tau_{, i} \tau_{, j} .
\end{aligned}
$$

We denoted by $\tau$ the thermal displacement related to the temperature variation. The relationship between $\tau$ and $\theta$ is given by

$$
\dot{\tau}=\theta \text {. }
$$

We consider the initial conditions

$$
\begin{aligned}
& u_{i}(x, 0)=u_{i}^{0}(x), \\
& \dot{u}_{i}(x, 0)=\dot{u}_{i}^{0}(x), \\
& \theta(x, 0)=\theta^{0}(x), \quad x \in \bar{B}, \\
& \varphi_{j}(x, 0)=\varphi_{j}^{0}(x), \quad x \in \bar{B}, \\
& \dot{\varphi}_{j}(x, 0)=\dot{\varphi}_{j}^{0}(x), \quad x \in \bar{B},
\end{aligned}
$$

and the boundary conditions

$$
\begin{aligned}
& u_{i}(x, t)=\tilde{u}_{i}(x, t) \quad \text { on } \bar{\Sigma}_{1} \times[0, \infty), \\
& t_{i}(x, t)=\tilde{t}_{i}(x, t) \quad \text { on } \Sigma_{2} \times[0, \infty), \\
& \theta(x, t)=\tilde{\theta}(x, t) \quad \text { on } \bar{\Sigma}_{3} \times[0, \infty), \\
& q(x, t)=\tilde{q}(x, t) \quad \text { on } \Sigma_{4} \times[0, \infty), \\
& \varphi_{j}(x, t)=\tilde{\varphi}_{j}(x, t) \quad \text { on } \bar{\Sigma}_{5} \times[0, \infty), \\
& m_{i}(x, t)=\tilde{m}_{i}(x, t) \quad \text { on } \Sigma_{6} \times[0, \infty),
\end{aligned}
$$

where $u_{i}^{0}, \dot{u}_{i}^{0}, \theta^{0}, \dot{\varphi}_{j}^{0}, \tilde{u}_{i}, \tilde{t}_{i}, \tilde{\theta}, \tilde{q}, \tilde{\varphi}_{j}$, and $\tilde{m}_{i}$ are prescribed functions. We have

$$
\begin{aligned}
& t_{i}(x, s):=t_{i j}(x, s) n_{j}(x), \\
& q(x, s):=q_{i}(x, s) n_{i}(x), \\
& m_{i}(x, s):=m_{i j}(x, s) n_{j}(x),
\end{aligned}
$$

where $n_{i}$ are the components of the outward unit normal vector to the boundary surface and $\Sigma_{1}, \Sigma_{2}, \Sigma_{3}, \Sigma_{4}, \Sigma_{5}$, and $\Sigma_{6}$ are subsurfaces of $\partial B$ such that $\bar{\Sigma}_{1} \cup \Sigma_{2}=\bar{\Sigma}_{3} \cup \Sigma_{4}=\bar{\Sigma}_{5} \cup$ $\Sigma_{6}=\partial B$ and $\Sigma_{1} \cap \Sigma_{2}=\Sigma_{3} \cap \Sigma_{4}=\Sigma_{5} \cap \Sigma_{6}=\varnothing$. 
Following [3], we impose some continuity conditions

$$
\begin{aligned}
& \{u, \varphi\} \in C^{1,2}, \\
& \left\{\varepsilon, \gamma, F_{i}, M_{i}, r\right\} \in C^{0,0}, \\
& \{t, m, q\} \in C^{1,0}, \\
& \theta \in C^{1,0}, \quad \eta \in C^{0,1}, \\
& \left\{\tilde{u}_{i}, \tilde{t}_{i}, \tilde{\varphi}_{j}, \tilde{m}_{i}, \tilde{\theta}, \tilde{q}\right\} \in C^{0,0} \quad \text { on } \partial B \times[0, \infty), \\
& \left\{u_{i}^{0}, \dot{u}_{i}^{0}, \varphi_{j}^{0}, \dot{\varphi}_{j}^{0}, \theta^{0}\right\} \in C^{0} \quad \text { on } \bar{B}, \\
& \left\{D_{i j}, E_{i j}, A_{i j m n}, B_{i j m n}, C_{i j m n}, c, K_{i j}, \rho, I_{i j}\right\} \in C^{0} .
\end{aligned}
$$

We use the symbol $C^{i, j}$ to denote the class of functions whose space partial derivatives of order up to and including $i$ and whose time derivatives of order up to and including $j$ are continuous. Such formal continuity requirements exist to any order as demanded by the existing expressions, unless otherwise stated. Furthermore, we need

$$
\begin{aligned}
& \theta \in C^{1,1} \quad \text { on } \bar{B} \times[0, \infty), \\
& E_{i j}, D_{i j} \in C^{1}(\bar{B}) .
\end{aligned}
$$

Following [3], we define an admissible state to be the collection $S\{u, \varphi ; \varepsilon, \gamma ; t, m ; q, \theta\}$ of the ordered set of functions $u, \varphi, \varepsilon, \gamma, t, m, q$, and $\theta$ whose continuity requirements are described above. If $S$ meets the constitutive equations (3) and the strain displacement requirements (4) then we say that $S$ is kinematically admissible. If a kinematically admissible state meets the boundary and initial conditions and satisfies the equations of motion (1) and the equation of energy (2), we call it the solution of the mixed problem.

Introducing the constitutive equations (3) and the geometric equations (4) in the equations of motion (1) and the equation of energy (2), we obtain a system of coupled partial differential equations in terms of the displacements $u_{i}$, the microrotations $\varphi_{i}$ and the thermal displacements $\theta$

$$
\begin{aligned}
& \quad\left[A_{i j m n}\left(u_{n, m}+\varepsilon_{n m k} \varphi_{k}\right)+B_{i j m n} \varphi_{n, m}-D_{i j} \theta\right]_{, j}+\rho F_{i}=\rho \ddot{u}_{i}, \\
& {\left[B_{m n i j}\left(u_{n, m}+\varepsilon_{n m k} \varphi_{k}\right)+C_{i j m n} \varphi_{n, m}-E_{i j} \theta\right]_{, j}} \\
& \quad+\varepsilon_{i j k}\left[A_{j k m n}\left(u_{n, m}+\varepsilon_{n m k} \varphi_{k}\right)+B_{j k m n} \varphi_{n, m}-D_{j k} \theta\right]+\rho M_{i}=I_{i j} \ddot{\varphi}_{j}, \\
& D_{i j}\left(\dot{u}_{j, i}+\varepsilon_{j i k} \dot{\varphi}_{k}\right)+E_{i j} \dot{\varphi}_{j, i}+\frac{c}{\theta_{0}} \dot{\theta}=\frac{\rho}{\theta_{0}} r+\frac{1}{\theta_{0}}\left(K_{i j} \theta_{j, j}\right)_{, i},
\end{aligned}
$$

for any $(x, t) \in B \times[0, \infty)$.

By a solution of the initial boundary value problem of the micropolar thermoelasticity in the cylinder $B \times[0, T)$ we mean an ordered array $\left\{u_{i}, \varphi_{i}, \theta\right\}$ which satisfies system (16) for all $(x, t) \in B \times[0, T)$, the initial conditions (9) and the boundary conditions (10).

\section{Some useful identities}

Throughout this paper it is assumed that a solution $\left\{u_{i}, \varphi_{i}, \theta\right\}$ exists. Following [10] and [1], we establish some auxiliary identities for the solution of the initial boundary value 
problem. We consider the external data

$$
\mathcal{D}=\left\{F_{i}, M_{i}, r ; u_{i}^{0}, \dot{u}_{i}^{0}, \theta^{0}, \varphi_{j}^{0}, \dot{\varphi}_{j}^{0} ; \tilde{u}_{i}, \tilde{t}_{i}, \tilde{\theta}, \tilde{q}, \tilde{\varphi}_{j}, \tilde{m}_{i}\right\} .
$$

Lemma 2.1 Let us consider a solution of the initial boundary value problem corresponding to the external data $\mathcal{D}$. Then for all $t \in[0, T)$ we have

$$
\begin{aligned}
& \int_{B} \rho \dot{u}_{i}(t) \dot{u}_{i}(t) \mathrm{d} v \\
& =\int_{B} \rho \dot{u}_{i}(0) \dot{u}_{i}(0) \mathrm{d} v+2 \int_{0}^{t} \int_{B} \rho F_{i}(s) \dot{u}_{i}(s) \mathrm{d} v \mathrm{~d} s \\
& +2 \int_{0}^{t} \int_{\partial B} \dot{u}_{i}(s) t_{i}(s) \mathrm{d} a \mathrm{~d} s+2 \int_{0}^{t} \int_{B} D_{i j} \theta(s) \dot{u}_{i, j}(s) \mathrm{d} v \mathrm{~d} s \\
& -2 \int_{0}^{t} \int_{B} A_{i j m n}\left[u_{n, m}(s)+\varepsilon_{n m k} \varphi_{k}(s)\right] \dot{u}_{i, j}(s) \mathrm{d} v \mathrm{~d} s \\
& -2 \int_{0}^{t} \int_{B} B_{i j m n} \varphi_{n, m}(s) \dot{u}_{i, j}(s) \mathrm{d} v \mathrm{~d} s, \\
& \int_{B} \frac{1}{\theta_{0}} c \theta^{2}(t) \mathrm{d} v+2 \int_{0}^{t} \int_{B} \frac{1}{\theta_{0}} K_{i j} \beta_{i}(s) \beta_{j}(s) \mathrm{d} v \mathrm{~d} s \\
& =2 \int_{0}^{t} \int_{B} \theta(s) \frac{\rho}{\theta_{0}} r(s) \mathrm{d} v \mathrm{~d} s \\
& -2 \int_{0}^{t} \int_{\partial B} \theta(s) q(s) \mathrm{d} a \mathrm{~d} s-2 \int_{0}^{t} \int_{B} \theta(s) D_{i j}\left[\dot{u}_{j, i}(s)+\varepsilon_{j i k} \dot{\varphi}_{k}(s)\right] \mathrm{d} v \mathrm{~d} s \\
& -2 \int_{0}^{t} \int_{B} \theta(s) E_{i j} \dot{\varphi}_{j, i}(s) \mathrm{d} v \mathrm{~d} s+\int_{B} \frac{1}{\theta_{0}} c \theta^{2}(0) \mathrm{d} v, \\
& \int_{B} I_{i j} \dot{\varphi}_{i}(t) \dot{\varphi}_{j}(t) \mathrm{d} v \\
& =\int_{B} I_{i j} \dot{\varphi}_{i}(0) \dot{\varphi}_{j}(0) \mathrm{d} v+2 \int_{0}^{t} \int_{\partial B} \dot{\varphi}_{i}(s) m_{i j}(s) n_{j} \mathrm{~d} a \mathrm{~d} s \\
& -2 \int_{0}^{t} \int_{B} B_{m n i j}\left[u_{n, m}(s)+\varepsilon_{n m k} \varphi_{k}(s)\right] \dot{\varphi}_{i, j}(s) \mathrm{d} \nu \mathrm{d} s \\
& +2 \int_{0}^{t} \int_{B} C_{i j m n} \varphi_{n, m}(s) \dot{\varphi}_{i, j}(s) \mathrm{d} v \mathrm{~d} s \\
& -2 \int_{0}^{t} \int_{B} E_{i j} \theta(s) \dot{\varphi}_{i, j}(s) \mathrm{d} v \mathrm{~d} s+2 \int_{0}^{t} \int_{B} \varepsilon_{i j k} A_{j k m n}\left[u_{n, m}(s)+\varepsilon_{n m k} \varphi_{k}(s)\right] \dot{\varphi}_{i}(s) \mathrm{d} v \mathrm{~d} s \\
& +2 \int_{0}^{t} \int_{B} \varepsilon_{i j k} B_{j k m n} \varphi_{n, m}(s) \dot{\varphi}_{i}(s) \mathrm{d} \nu \mathrm{d} s-2 \int_{0}^{t} \int_{B} \varepsilon_{i j k} D_{j k} \theta(s) \dot{\varphi}_{i}(s) \mathrm{d} \nu \mathrm{d} s \\
& +2 \int_{0}^{t} \int_{B} \rho M_{i} \dot{\varphi}_{i}(s) \mathrm{d} v \mathrm{~d} s
\end{aligned}
$$

Proof We multiply the first equation from (1) by $\dot{u}_{i}$ to obtain

$$
\rho \dot{u}_{i}(s) \ddot{u}_{i}(s)=\rho F_{i}(s) \dot{u}_{i}(s)+\left[t_{i j}(s) \dot{u}_{i}(s)\right]_{, j}-t_{i j}(s) \dot{u}_{i, j}(s) .
$$


We further have

$$
\begin{aligned}
\frac{\partial}{\partial s}\{ & \left.\frac{1}{2}\left[\rho \dot{u}_{i}(s) \dot{u}_{i}(s)\right]\right\} \\
= & \rho F_{i}(s) \dot{u}_{i}(s)+\left[t_{i j}(s) \dot{u}_{i}(s)\right]_{, j} \\
& -A_{i j m n}\left[u_{n, m}(s)+\varepsilon_{n m k} \varphi_{k}(s)\right] \dot{u}_{i, j}(s)-B_{i j m n} \varphi_{n, m}(s) \dot{u}_{i, j}(s)+D_{i j} \theta(s) \dot{u}_{i, j}(s) .
\end{aligned}
$$

We integrate this relation over $B \times[0, t], t \in[0, T)$, and use the divergence theorem and equation (11) to obtain equation (18).

We have

$$
\frac{\partial}{\partial s}\left\{\frac{1}{2} \frac{1}{\theta_{0}} c \theta^{2}(s)\right\}=\theta(s) \frac{1}{\theta_{0}} c \dot{\theta}(s) .
$$

We further have by the third relation in (3) and by (2)

$$
\frac{\partial}{\partial s}\left\{\frac{1}{2} \frac{1}{\theta_{0}} c \theta^{2}(s)\right\}=\theta(s) \frac{\rho}{\theta_{0}} r(s)-\theta(s) q_{i, i}(s)-\theta(s) D_{i j} \dot{\varepsilon}_{i j}(s)-\theta(s) E_{i j} \dot{\gamma}_{i j}(s),
$$

which by $\theta(s) q_{i, i}(s)=\left[\theta(s) q_{i}(s)\right]_{, i}-\theta_{, i}(s) q_{i}(s)$, by the fourth relation in (3), and by (5) becomes

$$
\begin{aligned}
\frac{\partial}{\partial s} & \left\{\frac{1}{2} \frac{1}{\theta_{0}} c \theta^{2}(s)\right\} \\
& =\theta(s) \frac{\rho}{\theta_{0}} r(s)-\left[\theta(s) q_{i}(s)\right]_{, i}-\beta_{i}(s) \frac{1}{\theta_{0}} K_{i j} \beta_{j}(s)-\theta(s) D_{i j} \dot{\varepsilon}_{i j}(s)-\theta(s) E_{i j} \dot{\gamma}_{i j}(s) .
\end{aligned}
$$

We integrate this relation over $B \times[0, t], t \in[0, T)$, and use the divergence theorem and equation (12) to obtain equation (19).

We multiply the second equation from (1) by $\dot{\varphi}_{i}$ to obtain

$$
I_{i j} \ddot{\varphi}_{j}(s) \dot{\varphi}_{i}(s)=\left[m_{i j}(s) \dot{\varphi}_{i}(s)\right]_{, j}-m_{i j}(s) \dot{\varphi}_{i, j}(s)+\varepsilon_{i j k} t_{j k}(s) \dot{\varphi}_{i}(s)+\rho M_{i} \dot{\varphi}_{i}(s)
$$

We further have

$$
\begin{aligned}
\frac{\partial}{\partial s}\{ & \left.\frac{1}{2}\left[I_{i j} \dot{\varphi}_{i}(s) \dot{\varphi}_{j}(s)\right]\right\} \\
= & {\left[m_{i j}(s) \dot{\varphi}_{i}(s)\right]_{, j}-\left[B_{m n i j} \varepsilon_{m n}(s)+C_{i j m n} \gamma_{m n}(s)-E_{i j} \theta(s)\right] \dot{\varphi}_{i, j}(s) } \\
& +\varepsilon_{i j k}\left[A_{j k m n} \varepsilon_{m n}(s)+B_{j k m n} \gamma_{m n}(s)-D_{j k} \theta(s)\right] \dot{\varphi}_{i}(s)+\rho M_{i} \dot{\varphi}_{i}(s) \\
= & {\left[m_{i j}(s) \dot{\varphi}_{i}(s)\right]_{, j}-B_{m n i j}\left[u_{n, m}(s)+\varepsilon_{n m k} \varphi_{k}(s)\right] \dot{\varphi}_{i, j}(s)+C_{i j m n} \varphi_{n, m}(s) \dot{\varphi}_{i, j}(s) } \\
& -E_{i j} \theta(s) \dot{\varphi}_{i, j}(s)+\varepsilon_{i j k} A_{j k m n}\left[u_{n, m}(s)+\varepsilon_{n m k} \varphi_{k}(s)\right] \dot{\varphi}_{i}(s) \\
& +\varepsilon_{i j k} B_{j k m n} \varphi_{n, m}(s) \dot{\varphi}_{i}(s)-\varepsilon_{i j k} D_{j k} \theta(s) \dot{\varphi}_{i}(s)+\rho M_{i} \dot{\varphi}_{i}(s) .
\end{aligned}
$$

We integrate this relation over $B \times[0, t], t \in[0, T)$, and use the divergence theorem and equation (13) to obtain equation (20). 
Lemma 2.2 Let us consider a solution of the initial boundary value problem corresponding to the external data $\mathcal{D}$. Then for all $t \in\left[0, \frac{T}{2}\right)$ we have

$$
\begin{aligned}
\int_{B} \rho \dot{u}_{i}(t) \dot{u}_{i}(t) \mathrm{d} v \\
=\int_{B} \rho \dot{u}_{i}(0) \dot{u}_{i}(2 t) \mathrm{d} v \\
\quad+\int_{0}^{t} \int_{B}\left[\rho F_{i}(t-s) \dot{u}_{i}(t+s)-\rho F_{i}(t+s) \dot{u}_{i}(t-s)\right] \mathrm{d} v \mathrm{~d} s \\
\quad+\int_{0}^{t} \int_{\partial B}\left[t_{i}(t-s) \dot{u}_{i}(t+s)-t_{i}(t+s) \dot{u}_{i}(t-s)\right] \mathrm{d} a \mathrm{~d} s \\
\quad+\int_{0}^{t} \int_{B}\left\{\dot{u}_{i, j}(t-s) A_{i j m n}\left[u_{n, m}+\varepsilon_{n m k} \varphi_{k}\right](t+s)\right. \\
\left.\quad-\dot{u}_{i, j}(t+s) A_{i j m n}\left[u_{n, m}+\varepsilon_{n m k} \varphi_{k}\right](t-s)\right\} \mathrm{d} v \mathrm{~d} s \\
\quad+\int_{0}^{t} \int_{B}\left[\dot{u}_{i, j}(t-s) B_{i j m n} \varphi_{n, m}(t+s)-\dot{u}_{i, j}(t+s) B_{i j m n} \varphi_{n, m}(t-s)\right] \mathrm{d} v \mathrm{~d} s \\
\quad+\int_{0}^{t} \int_{B}\left[\dot{u}_{i, j}(t+s) D_{i j} \theta(t-s)-\dot{u}_{i, j}(t-s) D_{i j} \theta(t+s)\right] \mathrm{d} v \mathrm{~d} s .
\end{aligned}
$$

Proof Let us consider $s \in[0, t], t \in\left[0, \frac{T}{2}\right)$. Then, using the identity

$$
-\frac{\partial}{\partial s}\left[\rho \dot{u}_{i}(t-s) \dot{u}_{i}(t+s)\right]=\rho \dot{u}_{i}(t+s) \ddot{u}_{i}(t-s)-\rho \dot{u}_{i}(t-s) \ddot{u}_{i}(t+s)
$$

we obtain

$$
\rho \dot{u}_{i}(t) \dot{u}_{i}(t)=\rho \dot{u}_{i}(0) \dot{u}_{i}(2 t)+\int_{0}^{t} \rho\left[\dot{u}_{i}(t+s) \ddot{u}_{i}(t-s)-\dot{u}_{i}(t-s) \ddot{u}_{i}(t+s)\right] \mathrm{d} s .
$$

By the first relation from (1), we have

$$
\begin{aligned}
& \rho\left[\dot{u}_{i}(t+s) \ddot{u}_{i}(t-s)-\dot{u}_{i}(t-s) \ddot{u}_{i}(t+s)\right] \\
&= \rho F_{i}(t-s) \dot{u}_{i}(t+s) \\
& \quad-\rho F_{i}(t+s) \dot{u}_{i}(t-s)+\left[t_{i j}(t-s) \dot{u}_{i}(t+s)-t_{i j}(t+s) \dot{u}_{i}(t-s)\right]_{, j} \\
& \quad-t_{i j}(t-s) \dot{u}_{i, j}(t+s)+t_{i j}(t+s) \dot{u}_{i, j}(t-s) .
\end{aligned}
$$

By the first relation in (3) and by (4) we have

$$
\begin{aligned}
t_{i j}(t+s) & \dot{u}_{i, j}(t-s)-t_{i j}(t-s) \dot{u}_{i, j}(t+s) \\
= & \dot{u}_{i, j}(t-s) A_{i j m n}\left[u_{n, m}+\varepsilon_{n m k} \varphi_{k}\right](t+s) \\
& -\dot{u}_{i, j}(t+s) A_{i j m n}\left[u_{n, m}+\varepsilon_{n m k} \varphi_{k}\right](t-s) \\
& +\dot{u}_{i, j}(t-s) B_{i j m n} \varphi_{n, m}(t+s)-\dot{u}_{i, j}(t+s) B_{i j m n} \varphi_{n, m}(t-s) \\
& -\dot{u}_{i, j}(t-s) D_{i j} \theta(t+s)+\dot{u}_{i, j}(t+s) D_{i j} \theta(t-s) .
\end{aligned}
$$


Now we substitute equation (32) in equation (31) and this in equation (30) to obtain

$$
\begin{aligned}
\rho \dot{u}_{i}(t) & \dot{u}_{i}(t) \\
= & \rho \dot{u}_{i}(0) \dot{u}_{i}(2 t)+\int_{0}^{t}\left[\rho F_{i}(t-s) \dot{u}_{i}(t+s)-\rho F_{i}(t+s) \dot{u}_{i}(t-s)\right] \mathrm{d} s \\
& +\int_{0}^{t}\left[t_{i j}(t-s) \dot{u}_{i}(t+s)-t_{i j}(t+s) \dot{u}_{i}(t-s)\right]_{, j} \mathrm{~d} s \\
& +\int_{0}^{t}\left\{\dot{u}_{i, j}(t-s) A_{i j m n}\left[u_{n, m}+\varepsilon_{n m k} \varphi_{k}\right](t+s)\right. \\
& \left.-\dot{u}_{i, j}(t+s) A_{i j m n}\left[u_{n, m}+\varepsilon_{n m k} \varphi_{k}\right](t-s)\right\} \mathrm{d} s \\
& +\int_{0}^{t}\left[\dot{u}_{i, j}(t-s) B_{i j m n} \varphi_{n, m}(t+s)-\dot{u}_{i, j}(t+s) B_{i j m n} \varphi_{n, m}(t-s)\right] \mathrm{d} s \\
& +\int_{0}^{t}\left[\dot{u}_{i, j}(t+s) D_{i j} \theta(t-s)-\dot{u}_{i, j}(t-s) D_{i j} \theta(t+s)\right] \mathrm{d} s .
\end{aligned}
$$

We integrate equation (33) over $B$, use the divergence theorem and equation (11) to obtain the final result.

\section{Zero external data}

Suppose that the boundary initial value problem of linear micropolar elastodynamics has two solutions $u^{(\alpha)}, \varphi^{(\alpha)}, \theta^{(\alpha)}, \alpha=1,2$. Let $u=u^{(1)}-u^{(2)}, \varphi=\varphi^{(1)}-\varphi^{(2)}, \theta=\theta^{(1)}-\theta^{(2)}$. Then $u, \varphi$, and $\theta$ satisfy (1)-(4), (9), and (10) with $F_{i}=M_{i}=0, r=0, \tilde{u}_{i}=\tilde{t}_{i}=\tilde{\theta}=\tilde{q}=\tilde{\varphi}_{j}=$ $\tilde{m}_{i}=0, u_{i}^{0}=\dot{u}_{i}^{0}=\theta^{0}=\varphi_{j}^{0}=\dot{\varphi}_{j}^{0}=0$, i.e. homogeneous equations and boundary and initial conditions.

Lemma 3.1 Let us consider a solution of the initial boundary value problem corresponding to zero external data $\mathcal{D}=0$. Then for all $t \in[0, T)$ we have

$$
\begin{aligned}
& \int_{B} \rho \dot{u}_{i}(t) \dot{u}_{i}(t) \mathrm{d} v \\
&= 2 \int_{0}^{t} \int_{B} D_{i j} \theta(s) \dot{u}_{i, j}(s) \mathrm{d} v \mathrm{~d} s \\
&-2 \int_{0}^{t} \int_{B} A_{i j m n}\left[u_{n, m}(s)+\varepsilon_{n m k} \varphi_{k}(s)\right] \dot{u}_{i, j}(s) \mathrm{d} v \mathrm{~d} s \\
&-2 \int_{0}^{t} \int_{B} B_{i j m n} \varphi_{n, m}(s) \dot{u}_{i, j}(s) \mathrm{d} v \mathrm{~d} s, \\
& \int_{B} \frac{1}{\theta_{0}} c \theta^{2}(t) \mathrm{d} v+2 \int_{0}^{t} \int_{B} \frac{1}{\theta_{0}} K_{i j} \beta_{i}(s) \beta_{j}(s) \mathrm{d} v \mathrm{~d} s \\
&=-2 \int_{0}^{t} \int_{B} \theta(s) D_{i j}\left[\dot{u}_{j, i}(s)+\varepsilon_{j i k} \dot{\varphi}_{k}(s)\right] \mathrm{d} v \mathrm{~d} s-2 \int_{0}^{t} \int_{B} \theta(s) E_{i j} \dot{\varphi}_{j, i}(s) \mathrm{d} v \mathrm{~d} s, \\
& \int_{B} I_{i j} \dot{\varphi}_{i}(t) \dot{\varphi}_{j}(t) \mathrm{d} v \\
&=-2 \int_{0}^{t} \int_{B}\left\{B_{m n i j}\left[u_{n, m}(s)+\varepsilon_{n m k} \varphi_{k}(s)\right]\right.
\end{aligned}
$$




$$
\begin{aligned}
& \left.+C_{i j m n} \varphi_{n, m}(s)-E_{i j} \theta(s)\right\} \dot{\varphi}_{i, j}(s) \mathrm{d} v \mathrm{~d} s \\
& +2 \int_{0}^{t} \int_{B} \varepsilon_{i j k}\left\{A_{j k m n}\left[u_{n, m}(s)+\varepsilon_{n m k} \varphi_{k}(s)\right]\right. \\
& \left.+B_{j k m n} \varphi_{n, m}(s)-D_{j k} \theta(s)\right\} \dot{\varphi}_{i}(s) \mathrm{d} v \mathrm{~d} s \\
\int_{B} \rho \dot{u}_{i} & (t) \dot{u}_{i}(t)+I_{i j} \dot{\varphi}_{i}(t) \dot{\varphi}_{j}(t)+\frac{1}{\theta_{0}} c \theta^{2}(t) \mathrm{d} v \\
= & -2 \int_{0}^{t} \int_{B}\left[u_{n, m}(s)+\varepsilon_{n m k} \varphi_{k}(s)\right]\left[A_{i j m n} \dot{u}_{i, j}(s)+B_{m n i j} \dot{\varphi}_{i, j}(s)\right. \\
& \left.-\varepsilon_{i j k} A_{j k m n} \dot{\varphi}_{i}(s)\right] \mathrm{d} v \mathrm{~d} s-2 \int_{0}^{t} \int_{B} \varphi_{n, m}(s)\left[B_{i j m n} \dot{u}_{i, j}(s)+C_{i j m n} \dot{\varphi}_{i, j}(s)\right. \\
& \left.-\varepsilon_{i j k} B_{j k m n} \dot{\varphi}_{i}(s)\right] \mathrm{d} v \mathrm{~d} s+2 \int_{0}^{t} \int_{B} D_{i j} \theta(s) \dot{u}_{i, j}(s) \mathrm{d} v \mathrm{~d} s+2 \int_{0}^{t} \int_{B} E_{i j} \theta(s) \dot{\varphi}_{i, j}(s) \mathrm{d} v \mathrm{~d} s \\
& -2 \int_{0}^{t} \int_{B} \varepsilon_{i j k} D_{j k} \theta(s) \dot{\varphi}_{i}(s) \mathrm{d} v \mathrm{~d} s-2 \int_{0}^{t} \int_{B} \theta(s) D_{i j}\left[\dot{u}_{j, i}(s)+\varepsilon_{j i k} \dot{\varphi}_{k}(s)\right] \mathrm{d} v \mathrm{~d} s \\
& -2 \int_{0}^{t} \int_{B} \theta(s) E_{i j} \dot{\varphi}_{j, i}(s) \mathrm{d} v \mathrm{~d} s-2 \int_{0}^{t} \frac{1}{\theta_{0}} K_{i j} \beta_{i}(s) \beta_{j}(s) \mathrm{d} v \mathrm{~d} s .
\end{aligned}
$$

Proof We choose $\dot{u}_{i}^{0}(x)=0, x \in \bar{B}, \tilde{u}_{i}(x, t)=0$ on $\bar{\Sigma}_{1} \times[0, \infty), \tilde{t}_{i}(x, t)=0$ on $\Sigma_{2} \times[0, \infty)$ and $F_{i}(x, t)=0$ on $\bar{B} \times[0, \infty)$ in (18) to obtain equation (34).

We choose $\theta^{0}(x)=0, x \in \bar{B}, \tilde{\theta}(x, t)=0$ on $\bar{\Sigma}_{3} \times[0, \infty), \tilde{q}(x, t)=0$ on $\Sigma_{4} \times[0, \infty)$ and $r(x, t)=0$ on $\bar{B} \times[0, \infty)$ in (19) to obtain equation (35).

We choose $\dot{\varphi}_{j}^{0}(x)=0, x \in \bar{B}, \tilde{\varphi}_{j}(x, t)=0$ on $\bar{\Sigma}_{5} \times[0, \infty), \tilde{m}_{i}(x, t)=0$ on $\Sigma_{6} \times[0, \infty)$ and $M_{i}(x, t)=0$ on $\bar{B} \times[0, \infty)$ in (20) to obtain equation (36).

We add up the previous three formulas.

Lemma 3.2 Let us consider a solution of the initial boundary value problem corresponding to zero external data $\mathcal{D}$. Then for all $t \in\left[0, \frac{T}{2}\right)$ we have

$$
\begin{aligned}
\int_{B} \rho \dot{u}_{i}(t) \dot{u}_{i}(t) \mathrm{d} v \\
=\int_{0}^{t} \int_{B}\left\{\dot{u}_{i, j}(t-s) A_{i j m n}\left[u_{n, m}+\varepsilon_{n m k} \varphi_{k}\right](t+s)\right. \\
\left.\quad-\dot{u}_{i, j}(t+s) A_{i j m n}\left[u_{n, m}+\varepsilon_{n m k} \varphi_{k}\right](t-s)\right\} \mathrm{d} v \mathrm{~d} s \\
\quad+\int_{0}^{t} \int_{B}\left[\dot{u}_{i, j}(t-s) B_{i j m n} \varphi_{n, m}(t+s)-\dot{u}_{i, j}(t+s) B_{i j m n} \varphi_{n, m}(t-s)\right] \mathrm{d} v \mathrm{~d} s \\
\quad+\int_{0}^{t} \int_{B}\left[\dot{u}_{i, j}(t+s) D_{i j} \theta(t-s)-\dot{u}_{i, j}(t-s) D_{i j} \theta(t+s)\right] \mathrm{d} v \mathrm{~d} s .
\end{aligned}
$$

Proof We choose $\dot{u}_{i}^{0}(x)=0, x \in \bar{B}, \tilde{u}_{i}(x, t)=0$ on $\bar{\Sigma}_{1} \times[0, \infty), \tilde{t}_{i}(x, t)=0$ on $\Sigma_{2} \times[0, \infty)$ and $F_{i}(x, t)=0$ on $\bar{B} \times[0, \infty)$ in (28) to obtain equation (38).

\section{Some useful remarks}

We assume that meas $\Sigma_{4}=0$. Let $\left\{u_{i}, \varphi_{j}, \theta\right\}(x, t)$ be a solution of the initial boundary value problem corresponding to zero external data $\mathcal{D}=0$. We might want to prove that 


$$
\begin{array}{r}
\left\{u_{i}, \varphi_{j}, \theta\right\}(x, t)=0 \text { in } \bar{B} \times[0, \infty) . \text { Let } \\
\phi(t)=\int_{0}^{t} \int_{B} \frac{1}{\theta_{0}} K_{i j} \beta_{i}(s) \beta_{j}(s) \mathrm{d} v \mathrm{~d} s
\end{array}
$$

for all $t \in[0, \infty)$. If $\phi(t)=0$ and $\theta_{0} \neq 0, K_{i j} \neq 0$, then either $\beta_{i}(x, s)=0$ or $\beta_{j}(x, s)=0$ in $B \times[0, \infty)$. If $\beta_{i}(x, s)=0$ then $\theta_{, i}(x, s)=0$ in $B \times[0, \infty)$. By [10] and [1] and since meas $\Sigma_{3} \neq 0$ and $\theta(x, t)=0$ on $\bar{\Sigma}_{3} \times[0, \infty)$ we have

$$
\int_{B} \theta_{, i}(t) \theta_{, i}(t) \mathrm{d} v \geq \lambda \int_{B} \theta^{2}(t) \mathrm{d} v
$$

where $\lambda>0, \lambda=$ const. is the smallest eigenvalue of the fixed membrane problem. Therefore $\theta(x, t)=0$ in $\bar{B} \times[0, \infty)$, which yields

$$
\begin{aligned}
& \int_{B} \rho \dot{u}_{i}(t) \dot{u}_{i}(t) \mathrm{d} v \\
&=-2 \int_{0}^{t} \int_{B} A_{i j m n}\left[u_{n, m}(s)+\varepsilon_{n m k} \varphi_{k}(s)\right] \dot{u}_{i, j}(s) \mathrm{d} v \mathrm{~d} s-2 \int_{0}^{t} \int_{B} B_{i j m n} \varphi_{n, m}(s) \dot{u}_{i, j}(s) \mathrm{d} v \mathrm{~d} s \\
&=-2 \int_{0}^{t} \int_{B} A_{i j m n} \varepsilon_{m n}(s) \dot{u}_{i, j}(s) \mathrm{d} v \mathrm{~d} s-2 \int_{0}^{t} \int_{B} B_{i j m n} \gamma_{m n}(s) \dot{u}_{i, j}(s) \mathrm{d} v \mathrm{~d} s \\
& \int_{B} I_{i j} \dot{\varphi}_{i}(t) \dot{\varphi}_{j}(t) \mathrm{d} v \\
&=-2 \int_{0}^{t} \int_{B}\left\{B_{m n i j}\left[u_{n, m}(s)+\varepsilon_{n m k} \varphi_{k}(s)\right]+C_{i j m n} \varphi_{n, m}(s)\right\} \dot{\varphi}_{i, j}(s) \mathrm{d} v \mathrm{~d} s \\
& \quad+2 \int_{0}^{t} \int_{B} \varepsilon_{i j k}\left\{A_{j k m n}\left[u_{n, m}(s)+\varepsilon_{n m k} \varphi_{k}(s)\right]+B_{j k m n} \varphi_{n, m}(s)\right\} \dot{\varphi}_{i, j}(s) \mathrm{d} v \mathrm{~d} s \\
&=-2 \int_{0}^{t} \int_{B}\left\{B_{m n i j} \varepsilon_{m n}(s)+C_{i j m n} \gamma_{m n}(s)\right\} \dot{\varphi}_{i, j}(s) \mathrm{d} v \mathrm{~d} s \\
&+2 \int_{0}^{t} \int_{B} \varepsilon_{i j k}\left\{A_{j k m n} \varepsilon_{m n}(s)+B_{j k m n} \gamma_{m n}(s)\right\} \dot{\varphi}_{i, j}(s) \mathrm{d} v \mathrm{~d} s .
\end{aligned}
$$

By imposing certain conditions on the coefficients, we might prove that $\varphi(x, t)=0$ and $u(x, t)=0$ in $\bar{B} \times[0, \infty)$. Following [3], we know that

$$
A_{k l m n} \varepsilon_{k l} \varepsilon_{m n}+C_{k l m n} \gamma_{k l} \gamma_{m n}+2 B_{k l m n} \varepsilon_{k l} \gamma_{m n} \geq 0
$$

Now we derive some useful inequalities for the constitutive coefficients $E_{i j}$ and $D_{i j}$. Since $\theta(x, s)=0$ on $\partial B \times[0, T)$ we have

$$
\begin{aligned}
- & 2 \int_{0}^{t} \int_{B} \theta(s) E_{i j} \dot{\varphi}_{j, i}(s) \mathrm{d} v \mathrm{~d} s \\
& =-2 \int_{0}^{t} \int_{B}\left\{\left[E_{i j} \theta(s) \dot{\varphi}_{j}(s)\right]_{, i}-\left[E_{i j} \theta(s)\right]_{, i} \dot{\varphi}_{j}(s)\right\} \mathrm{d} v \mathrm{~d} s \\
& =-2 \int_{0}^{t} \int_{\partial B} E_{i j} \theta(s) \dot{\varphi}_{j}(s) n_{i} \mathrm{~d} a \mathrm{~d} s+2 \int_{0}^{t} \int_{B}\left[E_{i j, i} \theta(s) \dot{\varphi}_{j}(s)+E_{i j} \theta_{, i}(s) \dot{\varphi}_{j}(s)\right] \mathrm{d} v \mathrm{~d} s \\
& =2 \int_{0}^{t} \int_{B}\left\{\left[\frac{\sqrt{\varepsilon_{1}}}{\sqrt{\rho_{0}}} E_{i j, i} \theta(s)\right]\left[\frac{\sqrt{\rho_{0}}}{\sqrt{\varepsilon_{1}}} \dot{\varphi}_{j}(s)\right]+\left[\frac{\sqrt{\varepsilon_{2}}}{\sqrt{\rho_{0}}} E_{i j} \theta_{, i}(s)\right]\left[\frac{\sqrt{\rho_{0}}}{\sqrt{\varepsilon_{2}}} \dot{\varphi}_{j}(s)\right]\right\} \mathrm{d} v \mathrm{~d} s,
\end{aligned}
$$


which by the Cauchy-Schwartz inequality and by the arithmetic-geometric mean inequality is bounded by

$$
\begin{aligned}
\leq & 2 \int_{0}^{t} \int_{B}\left\{\left[\frac{\sqrt{\varepsilon_{1}}}{\sqrt{\rho_{0}}} E_{i j, i} \theta(s)\right]^{2}\right. \\
& \left.+\left[\frac{\sqrt{\varepsilon_{2}}}{\sqrt{\rho_{0}}} E_{i j} \theta_{, i}(s)\right]^{2}\right\}^{\frac{1}{2}}\left\{\left[\frac{\sqrt{\rho_{0}}}{\sqrt{\varepsilon_{1}}} \dot{\varphi}_{j}(s)\right]^{2}+\left[\frac{\sqrt{\rho_{0}}}{\sqrt{\varepsilon_{2}}} \dot{\varphi}_{j}(s)\right]^{2}\right\}^{\frac{1}{2}} \mathrm{~d} \nu \mathrm{d} s \\
\leq & \int_{0}^{t} \int_{B}\left\{\left[\frac{\sqrt{\varepsilon_{1}}}{\sqrt{\rho_{0}}} E_{i j, i} \theta(s)\right]^{2}\right. \\
& \left.+\left[\frac{\sqrt{\varepsilon_{2}}}{\sqrt{\rho_{0}}} E_{i j} \theta_{, i}(s)\right]^{2}+\rho_{0} \dot{\varphi}_{j}(s) \dot{\varphi}_{j}(s)\left(\frac{1}{\varepsilon_{1}}+\frac{1}{\varepsilon_{2}}\right)\right\} \mathrm{d} \nu \mathrm{d} s .
\end{aligned}
$$

We assume that

$$
\begin{aligned}
& m_{E}=\sup _{\bar{B}}\left(E_{i j} E_{i j}\right)^{\frac{1}{2}}>0, \\
& m_{E}^{*}=\sup _{\bar{B}}\left(E_{i j, i} E_{k j, k}\right)^{\frac{1}{2}}>0 .
\end{aligned}
$$

We denote $Q_{j}^{E}=E_{i j} \theta_{, i}$ and

$$
Q_{j}^{E} Q_{j}^{E}=E_{i j} \theta_{, i} Q_{j}^{E} \leq\left(E_{i j} E_{i j}\right)^{\frac{1}{2}}\left(Q_{j}^{E} \theta_{, i} Q_{j}^{E} \theta_{, i}\right)^{\frac{1}{2}},
$$

which yields

$$
E_{i j} \theta_{, i} E_{k j} \theta_{, k} \leq m_{E}^{2} \theta_{, i} \theta_{, i}
$$

Therefore $\forall \varepsilon_{1}, \varepsilon_{2}>0$

$$
\begin{aligned}
& -2 \int_{0}^{t} \int_{B} \theta(s) E_{i j} \dot{\varphi}_{j, i}(s) \mathrm{d} \nu \mathrm{d} s \\
& \quad \leq \int_{0}^{t} \int_{B}\left\{\left(\frac{1}{\varepsilon_{1}}+\frac{1}{\varepsilon_{2}}\right) \rho_{0} \dot{\varphi}_{j}(s) \dot{\varphi}_{j}(s)+\frac{\varepsilon_{1}}{\rho_{0}} m_{E}^{* 2} \theta^{2}(s)+\frac{\varepsilon_{2}}{\rho_{0}} m_{E}^{2} \theta_{, i}(s) \theta_{, i}(s)\right\} \mathrm{d} v \mathrm{~d} s,
\end{aligned}
$$

which by (40) is further bounded by

$$
\leq \int_{0}^{t} \int_{B}\left\{\left(\frac{1}{\varepsilon_{1}}+\frac{1}{\varepsilon_{2}}\right) \rho_{0} \dot{\varphi}_{j}(s) \dot{\varphi}_{j}(s)+\left(\frac{\varepsilon_{1}}{\rho_{0}} m_{E}^{* 2} \lambda^{-1}+\frac{\varepsilon_{2}}{\rho_{0}} m_{E}^{2}\right) \theta_{, i}(s) \theta_{, i}(s)\right\} \mathrm{d} v \mathrm{~d} s .
$$

Since $\theta(x, t)=0$ in $\bar{B} \times[0, \infty)$, by (35) and by (51) we obtain

$$
\int_{0}^{t} \int_{B} \rho_{0} \dot{\varphi}_{j}(s) \dot{\varphi}_{j}(s) \mathrm{d} v \mathrm{~d} s \geq 0
$$

Hence we do not need any condition on $\rho_{0}$ to prove the positivity of the integral above. 
We perform similar computations for the constitutive coefficient $D_{i j}$. We have, since $\theta(x, s)=0$ on $\partial B \times[0, T)$,

$$
\begin{aligned}
- & 2 \int_{0}^{t} \int_{B} \theta(s) D_{i j} \dot{u}_{j, i}(s) \mathrm{d} \nu \mathrm{d} s \\
& =-2 \int_{0}^{t} \int_{B}\left\{\left[D_{i j} \theta(s) \dot{u}_{j}(s)\right]_{, i}-\left[D_{i j} \theta(s)\right]_{, i} \dot{u}_{j}(s)\right\} \mathrm{d} v \mathrm{~d} s \\
& =-2 \int_{0}^{t} \int_{\partial B} D_{i j} \theta(s) \dot{u}_{j}(s) n_{i} \mathrm{~d} a \mathrm{~d} s+2 \int_{0}^{t} \int_{B}\left[D_{i j, i} \theta(s) \dot{u}_{j}(s)+D_{i j} \theta_{, i}(s) \dot{u}_{j}(s)\right] \mathrm{d} v \mathrm{~d} s \\
& =2 \int_{0}^{t} \int_{B}\left\{\left[\frac{\sqrt{\varepsilon_{1}}}{\sqrt{\rho_{0}}} D_{i j, i} \theta(s)\right]\left[\frac{\sqrt{\rho_{0}}}{\sqrt{\varepsilon_{1}}} \dot{u}_{j}(s)\right]+\left[\frac{\sqrt{\varepsilon_{2}}}{\sqrt{\rho_{0}}} D_{i j} \theta_{, i}(s)\right]\left[\frac{\sqrt{\rho_{0}}}{\sqrt{\varepsilon_{2}}} \dot{u}_{j}(s)\right]\right\} \mathrm{d} v \mathrm{~d} s,
\end{aligned}
$$

which by the Cauchy-Schwartz inequality and by the arithmetic-geometric mean inequality is bounded by

$$
\begin{aligned}
\leq & 2 \int_{0}^{t} \int_{B}\left\{\left[\frac{\sqrt{\varepsilon_{1}}}{\sqrt{\rho_{0}}} D_{i, j i} \theta(s)\right]^{2}\right. \\
& \left.+\left[\frac{\sqrt{\varepsilon_{2}}}{\sqrt{\rho_{0}}} D_{i j} \theta_{, i}(s)\right]^{2}\right\}^{\frac{1}{2}}\left\{\left[\frac{\sqrt{\rho_{0}}}{\sqrt{\varepsilon_{1}}} \dot{u}_{j}(s)\right]^{2}+\left[\frac{\sqrt{\rho_{0}}}{\sqrt{\varepsilon_{2}}} \dot{u}_{j}(s)\right]^{2}\right\}^{\frac{1}{2}} \mathrm{~d} v \mathrm{~d} s \\
\leq & \int_{0}^{t} \int_{B}\left\{\left[\frac{\sqrt{\varepsilon_{1}}}{\sqrt{\rho_{0}}} D_{i, j i} \theta(s)\right]^{2}+\left[\frac{\sqrt{\varepsilon_{2}}}{\sqrt{\rho_{0}}} D_{i j} \theta_{i, i}(s)\right]^{2}+\rho_{0} \dot{u}_{j}(s) \dot{u}_{j}(s)\left(\frac{1}{\varepsilon_{1}}+\frac{1}{\varepsilon_{2}}\right)\right\} \mathrm{d} v \mathrm{~d} s .
\end{aligned}
$$

We assume that

$$
\begin{aligned}
& m_{D}=\sup _{\bar{B}}\left(D_{i j} D_{i j}\right)^{\frac{1}{2}}>0, \\
& m_{D}^{*}=\sup _{\bar{B}}\left(D_{i j, i} D_{k j, k}\right)^{\frac{1}{2}}>0 .
\end{aligned}
$$

We denote $Q_{j}^{D}=D_{i j} \theta_{, i}$ and

$$
Q_{j}^{D} Q_{j}^{D}=D_{i j} \theta_{, i} Q_{j}^{D} \leq\left(D_{i j} D_{i j}\right)^{\frac{1}{2}}\left(Q_{j}^{D} \theta_{, i} Q_{j}^{D} \theta_{, i}\right)^{\frac{1}{2}},
$$

which yields

$$
D_{i j} \theta_{, i} D_{k j} \theta_{, k} \leq m_{D}^{2} \theta_{, i} \theta_{i}
$$

Therefore $\forall \varepsilon_{1}, \varepsilon_{2}>0$

$$
\begin{aligned}
& -2 \int_{0}^{t} \int_{B} \theta(s) D_{i j} \dot{u}_{j, i}(s) \mathrm{d} v \mathrm{~d} s \\
& \quad \leq \int_{0}^{t} \int_{B}\left\{\left(\frac{1}{\varepsilon_{1}}+\frac{1}{\varepsilon_{2}}\right) \rho_{0} \dot{u}_{j}(s) \dot{u}_{j}(s)+\frac{\varepsilon_{1}}{\rho_{0}} m_{D}^{* 2} \theta^{2}(s)+\frac{\varepsilon_{2}}{\rho_{0}} m_{D}^{2} \theta_{, i}(s) \theta_{, i}(s)\right\} \mathrm{d} v \mathrm{~d} s,
\end{aligned}
$$

which by (40) is further bounded by

$$
\leq \int_{0}^{t} \int_{B}\left\{\left(\frac{1}{\varepsilon_{1}}+\frac{1}{\varepsilon_{2}}\right) \rho_{0} \dot{u}_{j}(s) \dot{u}_{j}(s)+\left(\frac{\varepsilon_{1}}{\rho_{0}} m_{D}^{* 2} \lambda^{-1}+\frac{\varepsilon_{2}}{\rho_{0}} m_{D}^{2}\right) \theta_{, i}(s) \theta_{, i}(s)\right\} \mathrm{d} v \mathrm{~d} s .
$$


Since $\theta(x, t)=0$ in $\bar{B} \times[0, \infty)$, by (35) and by (60) we obtain

$$
\int_{0}^{t} \int_{B} \rho_{0} \dot{u}_{j}(s) \dot{u}_{j}(s) \mathrm{d} v \mathrm{~d} s \geq 0
$$

Hence we do not need any condition on $\rho_{0}$ to prove the positivity of the integral above.

We say that $K_{i j}$ is a positive definite tensor if there exists a positive constant $k_{0}>0$ such that $\forall \xi_{i}$

$$
K_{i j} \xi_{i} \xi_{j} \geq k_{0} \xi_{i} \xi_{i}
$$

Thus $k_{0}$ can be identified with the minimum of the positive eigenvalues of $K_{i j}$ on $\bar{B}$. Since $K_{i j} \theta_{, i} \theta_{, j} \geq k_{0} \theta_{, i} \theta_{, i}$ we have $\forall \varepsilon_{1}, \varepsilon_{2}>0, t \in[0, T)$

$$
\begin{aligned}
-2 & \int_{0}^{t} \int_{B} \theta(s) E_{i j} \dot{\varphi}_{j, i}(s) \mathrm{d} \nu \mathrm{d} s \\
\leq & \left(\frac{1}{\varepsilon_{1}}+\frac{1}{\varepsilon_{2}}\right) \int_{0}^{t} \int_{B} \rho_{0} \dot{\varphi}_{j}(s) \dot{\varphi}_{j}(s) \mathrm{d} \nu \mathrm{d} s \\
& +\frac{\theta_{0}}{\rho_{0} k_{0}}\left(\varepsilon_{1} m_{E}^{* 2} \lambda^{-1}+\varepsilon_{2} m_{E}^{2}\right) \int_{0}^{t} \int_{B} \frac{1}{\theta_{0}} K_{i j} \theta_{, i}(s) \theta_{, j}(s) \mathrm{d} \nu \mathrm{d} s
\end{aligned}
$$

and

$$
\begin{aligned}
-2 & \int_{0}^{t} \int_{B} \theta(s) D_{i j} \dot{u}_{j, i}(s) \mathrm{d} \nu \mathrm{d} s \\
\leq & \left(\frac{1}{\varepsilon_{1}}+\frac{1}{\varepsilon_{2}}\right) \int_{0}^{t} \int_{B} \rho_{0} \dot{u}_{j}(s) \dot{u}_{j}(s) \mathrm{d} \nu \mathrm{d} s \\
& +\frac{\theta_{0}}{\rho_{0} k_{0}}\left(\varepsilon_{1} m_{D}^{* 2} \lambda^{-1}+\varepsilon_{2} m_{D}^{2}\right) \int_{0}^{t} \int_{B} \frac{1}{\theta_{0}} K_{i j} \theta_{, i}(s) \theta_{, j}(s) \mathrm{d} \nu \mathrm{d} s .
\end{aligned}
$$

We choose parameters $\varepsilon_{1}, \varepsilon_{2}$ so small that

$$
\alpha=2-\frac{\theta_{0}}{\rho_{0} k_{0}}\left(\varepsilon_{1} m_{D}^{* 2} \lambda^{-1}+\varepsilon_{2} m_{D}^{2}\right)-\frac{\theta_{0}}{\rho_{0} k_{0}}\left(\varepsilon_{1} m_{E}^{* 2} \lambda^{-1}+\varepsilon_{2} m_{E}^{2}\right)>0 .
$$

Hence from equation (35) we have

$$
\begin{aligned}
\int_{B} & \frac{1}{\theta_{0}} c \theta^{2}(t) \mathrm{d} v+\alpha \int_{0}^{t} \int_{B} \frac{1}{\theta_{0}} K_{i j} \beta_{i}(s) \beta_{j}(s) \mathrm{d} v \mathrm{~d} s \\
\leq & \left(\frac{1}{\varepsilon_{1}}+\frac{1}{\varepsilon_{2}}\right) \int_{0}^{t} \int_{B} \rho_{0} \dot{u}_{j}(s) \dot{u}_{j}(s) \mathrm{d} v \mathrm{~d} s \\
& +\left(\frac{1}{\varepsilon_{1}}+\frac{1}{\varepsilon_{2}}\right) \int_{0}^{t} \int_{B} \rho_{0} \dot{\varphi}_{j}(s) \dot{\varphi}_{j}(s) \mathrm{d} v \mathrm{~d} s-2 \int_{0}^{t} \int_{B} \theta(s) D_{i j} \varepsilon_{i j k} \varphi_{k}(s) \mathrm{d} \nu \mathrm{d} s .
\end{aligned}
$$

The authors declare that they have no competing interests. 
Author details

'Department of Mathematics, Transilvania University of Braşov, Braşov, Romania. ${ }^{2}$ Department of Mathematics, Texas A\&M University - Kingsville, Kingsville, USA.

\section{Acknowledgements}

The authors would like to thank the reviewers for valuable comments and suggestions.

Received: 8 November 2016 Accepted: 18 December 2016 Published online: 03 January 2017

\section{References}

1. Chiriţă, S: On the final boundary value problems in linear thermoelasticity. Meccanica 47, 2005-2011 (2012)

2. Marin, M, Baleanu, D: On vibrations in thermoelasticity without energy dissipation for micropolar bodies. Bound. Value Probl. 2016, 111 (2016). doi:10.1186/s13661-016-0620-9

3. Eringen, AC: Microcontinuum Field Theories: Foundations and Solids. Springer, New York (1998)

4. Ciarletta, M: A theory of micropolar thermoelasticity without energy dissipation. J. Therm. Stresses 22, $581-594$ (1999)

5. Ieşan, D: Some applications of micropolar mechanics to earthquake problems. Int. J. Eng. Sci. 19, $855-864$ (1981)

6. Marin, M: On existence and uniqueness in thermoelasticity of micropolar bodies. C. R. Math. Acad. Sci. Paris Ser. II $321(12), 475-480$ (1995)

7. Marin, M: Some basic results in elastostatics of micropolar materials with voids. J. Comput. Appl. Math. 70(1), 115-126 (1996)

8. Marin, M, Marinescu, C: Thermoelasticity of initially stressed bodies. Asymptotic equipartition of energies. Int. J. Eng. Sci. 36(1), 73-86 (1998)

9. Ieşan, D: Mecanica generalizată a solidelor, Universitatea "Al. I. Cuza", Centrul de multiplicare, lasi (1980) 404 pp.

10. Ciarletta, $\mathrm{M}$ : On the uniqueness and continuous dependence of solutions in dynamical thermoelasticity backward in time. J. Therm. Stresses 25, 969-984 (2002)

\section{Submit your manuscript to a SpringerOpen ${ }^{\circ}$ journal and benefit from:}

- Convenient online submission

Rigorous peer review

- Immediate publication on acceptance

- Open access: articles freely available online

- High visibility within the field

- Retaining the copyright to your article 